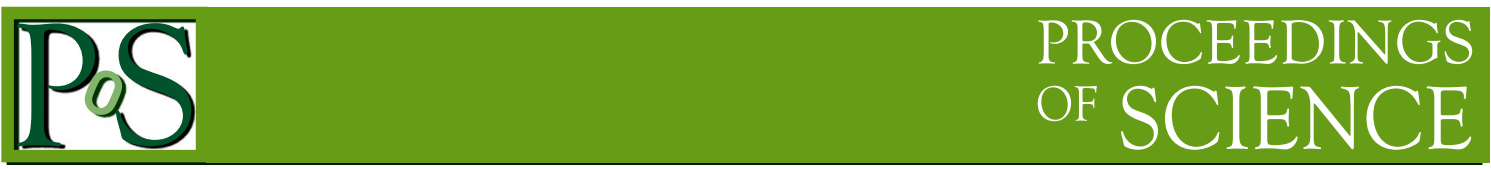

\title{
Nuclear Astrophysics and LUNA MV
}

\author{
Alessandra Guglielmetti ${ }^{1}$ for the LUNA collaboration \\ Università degli Studi di Milano and INFN Milano \\ Via Celoria 1620133 Milano, Italy \\ E-mail: alessandra.guglielmettiemi.infn.it
}

Nuclear astrophysics is an extremely rich field, strongly correlated with many other research fields like observational astronomy, stellar modelling, neutrino physics, cosmology, nuclear physics etc. One of the most ambitious task of nuclear astrophysics is to explain the origin and relative abundances of the elements in the Universe, formed through different nuclear processes in different astrophysical scenarios. Therefore, nuclear fusion reactions are the core of nuclear astrophysics since they determine the formation of the elements in the earliest stages of the Universe (Big Bang nucleosynthesis, BBN) and in all the objects formed thereafter and control the energy generation, neutrino production and evolution of stars. After 25 years of measurements on the cross section of key reactions belonging to BBN or Hydrogen burning, the LUNA collaboration is going to start a new ambitious program devoted to Helium and Carbon burning using the new 3.5 MV ñLUNA MVò accelerator which will be installed in the underground laboratory of Gran Sasso next year. After a general introduction on nuclear astrophysics and underground techniques, the scientific goals of the LUNA MV project for the first five years and its present status will be described.

XVII International Workshop on Neutrino Telescopes

13-17 March 2017

Venezia, Italy

\section{${ }^{1}$ Speaker}




\section{Nuclear astrophysics: general features, needs and techniques: The LUNA approach}

Nuclear astrophysics is based on accurate and precise knowledge of the cross sections of thermonuclear fusion reactions occurring during BBN and in all burning stages of different stellar objects. Such primary ingredients are thus very important at first for BBN, with all its cosmological implications, but also for stellar models and in general for describing the evolution of the Universe. Despite the big experimental efforts of the last fifty years or more, many reactions still ask for high precision data. Moreover, the precise knowledge of reactions producing neutrinos is mandatory to use neutrinos as probes of the stellar interior and to derive information on the particleâ properties. From the nuclear physics point of view, the structure of the involved nuclei play a very important role in determining the reaction mechanism, which is obviously related with the reaction probability (cross section).

Thermonuclear fusion reactions occur in a very well defined energy range, the so-called Gamow peak [1], which arises from the convolution of the energy distribution of nuclei in the stellar plasma and the tunnelling probability through the Coulomb barrier between the interacting charged particles. In a non-degenerate, non-relativistic stellar plasma, the former is given by the Maxwell-Boltzmann distribution, with a maximum for $\mathrm{E}=\mathrm{kT}$ (where $\mathrm{T}$ is the star temperature and $\mathrm{k}$ the Maxwell-Boltzmann constant) and then decreases exponentially with increasing energy, while the latter decreases exponentially for decreasing energy. For example, for a central temperature of $1.510^{7} \mathrm{~K}$, as in our Sun, the maximum of the Maxwell-Boltzmann distribution occurs at about $1 \mathrm{keV}$, while the Coulomb barrier for most reactions in either the p-p chain or the CNO cycle of Hydrogen burning is between 0.5 and $2 \mathrm{MeV}$. The Gamow peak for the same reactions is below $30 \mathrm{keV}$ and, as a result, reaction cross sections can be extremely low, down to the femto-barn level, due to the already mentioned exponential drop of the tunnelling probability with decreasing energy. It follows that a direct investigation of thermonuclear reactions at or near their Gamow energy is often beyond technical capabilities as the signal-to-noise ratio is severely dominated by any source of unwanted background. This can be given by the environmental background from $\mathrm{U}$ and $\mathrm{Th}$ radioactive chains and from ${ }^{40} \mathrm{~K}$, by the beam induced background from parasitic reactions with impurities in the experimental apparatus and by the cosmic background. In a laboratory at the Earthô surface, the greatest contribution is typically given by such third component, precisely by the interaction of cosmic rays with materials in the detection setup. Reaction cross-sections are thus extrapolated from data taken at higher energies, although significant uncertainties at the lowest energies of astrophysical interest remain. As a matter of fact, the existence of narrow resonances in or near the Gamow energy region, the tails of broad resonances and/or sub-threshold states, as well as a change in the reaction mechanism at ultra-low energies, can all translate in non-negligible contributions to the reaction cross section (or equivalently the astrophysical factor) thus rendering the extrapolation procedure extremely uncertain.

An alternative solution requires a drastic reduction of any unwanted background to optimise the signal-to-noise ratio. This can often be achieved by carrying out the measurement in a deep underground laboratory. The LUNA (Laboratory for Underground Nuclear Astrophysics) collaboration has exploited the low-background environment of the underground laboratory under the Gran Sasso Mountain in Italy (LNGS) to perform direct measurements at the relevant 
astrophysical energies. The rock overburden of about $1400 \mathrm{~m}$ (3800 m water equivalent) reduces the muon component of the cosmic background by a factor of $10^{6}$; the neutron component by a factor of $10^{3}$; and the gamma component by a factor of 10 with respect to a laboratory on the Earthô surface. As a result, the gamma background above $3 \mathrm{MeV}$ in an HPGe detector placed underground at LNGS is reduced by a factor of $\sim 2500$ with respect to the same detector placed over-ground. In addition, going underground enhances the effect of passive shielding used to reduce the contribution of low energy $(<3 \mathrm{MeV})$ gammas due to environmental radioactivity. Indeed, a passive shield can be built around the detector also in a laboratory at the Earthô surface. However, the shielding efficiency cannot be increased ad libitum by further adding more shield since the cosmic muons would interact with the added material, creating more background. This problem is of course dramatically reduced in an underground laboratory. Here, an extremely large thickness can be adopted with a consequent strong reduction of the environmental background. Finally, a factor 15 background suppression with respect to overground for the detection of 200 $\mathrm{keV}$ alpha particles with silicon detectors can be achieved [2].

The LUNA collaboration has installed two accelerators underground: a compact $50 \mathrm{kV}$ ñhome-madeò machine [3] and a commercial $400 \mathrm{kV}$ one [4]. Common features of the two are the intense beam current achievable, the long-term stability, and the precise energy determination. The first two features are essential to maximize the reaction rate, while the third one is important in view of the exponential energy dependence of the cross section. With the first machine, operating between 1992 and 2001, two key reactions of the p-p chain were studied at the solar Gamow peak energies and the screening effect, i.e. the lowering of the Coulomb barrier for a nucleus surrounded by electrons with respect to a bare one, was investigated. The $400 \mathrm{kV}$ machine started operations in the year 2000 and is still operating: a few key reactions of the CNO, NeNa and $\mathrm{MgAl}$ cycles of Hydrogen burning, and of BBN were successfully measured with important astrophysical and cosmological consequences.

The most important results obtained by the LUNA collaboration with both machines are included in two reviews $[5,6]$ and in the topical issue dedicated to underground nuclear astrophysics and solar neutrinos [7]. Still, a few reactions are being or will be measured in the next 4-5 years. Nevertheless, the big step forward in the field will be given by the LUNA MV project.

\section{The LUNA MV project: scientific goals and present status}

In 2018, the installation of a new, 3.5 MV, accelerator inside the Hall B of LNGS laboratory is expected. The project has been financed in the framework of the òProgetti Premialiò calls of the Italian Research Ministry (years 2011 and 2012) with a total budget of about 5.3 millions of euros. The machine will be able to provide intense beams of $\mathrm{H}^{+},{ }^{4} \mathrm{He}^{+},{ }^{12} \mathrm{C}^{+} \mathrm{e}^{12} \mathrm{C}^{++}$in the energy range: $350 \mathrm{keV}-3.5 \mathrm{MeV}$. Two different beam lines are foreseen, devoted to solid and gas target experiments, respectively. This will allow the preparation of the experimental setup on one beam line while running a measurement on the other beam line. The accelerator room will have $80 \mathrm{~cm}$ thick concrete walls and ceiling, working as neutron shielding. The maximum neutron flux just outside the shielding will be, on average, a factor of twenty smaller than the natural neutron background at LNGS: this will prevent any interference with all the other activities running at the Laboratory. A sketch of the experimental hall is given in Figure 1. 


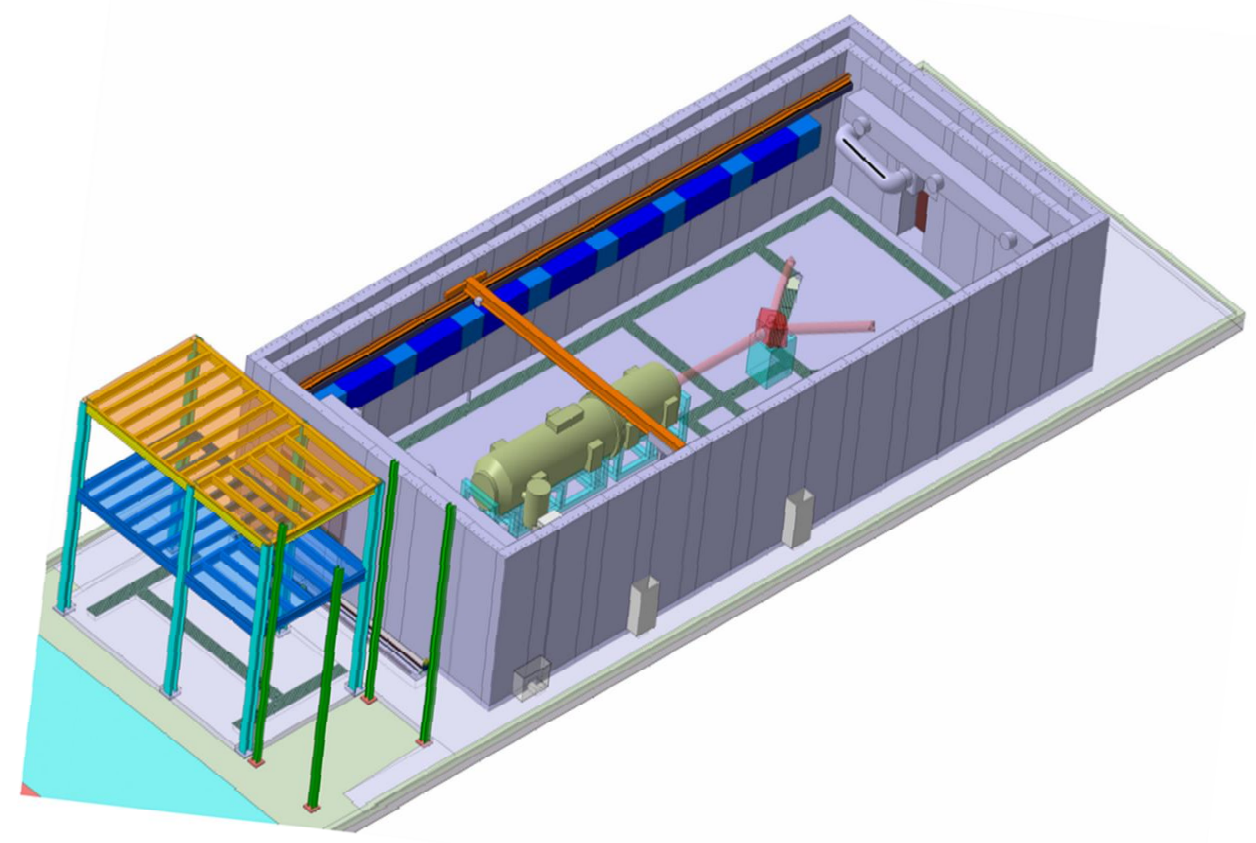

Figure 1: Sketch of the LUNA MV experimental hall

The program for the first five years of scientific activities has been outlined. At first the ${ }^{14} \mathrm{~N}(\mathrm{p}, \gamma){ }^{15} \mathrm{O}$ reaction will be measured in a wide energy range. In fact, despite all the measurements performed on this reaction by different groups, the status of the art is still unsatisfactory: in particular, the ground state transition is affected by a large systematic uncertainty. The scientific interest is connected to the problem of the solar composition: the conflict between the helioseismology results and the predictions of the Standard Solar Model once the new value of the metal abundance (i.e. the amount of elements different from hydrogen and helium) that emerged from improved modeling of the solar photosphere are used. In the near future the first measurement of the CNO neutrinos from the Sun will be accomplished. At that moment, the carbon and nitrogen content of the Sun core will be obtained from the comparison between the measured CNO neutrino flux and the predicted one. As a matter of fact, the CNO neutrino flux depends linearly both on the ${ }^{14} \mathrm{~N}(\mathrm{p}, \gamma){ }^{15} \mathrm{O}$ cross section and on the carbon and nitrogen abundance and it decreases by about $30 \%$ in going from the high to the low metallicity scenario. The ${ }^{14} \mathrm{~N}(\mathrm{p}, \gamma){ }^{15} \mathrm{O}$ measurement will serve also as commissioning experiment for the LUNA MV facility, being the techniques to produce the targets and detect the gammas already well known by the LUNA collaboration.

The flagship reaction for the first five-year scientific program is the ${ }^{12} \mathrm{C}+{ }^{12} \mathrm{C}$. This is the fundamental reaction of the Carbon burning influencing the global chemical evolution of the Universe. In fact, the end of helium burning marks a branching point in stellar evolution. Lower mass stars will become stable, electron-degenerate white dwarfs, while higher mass stars will enter the quiescent carbon burning phase of their evolution. Therefore, this limit separates the progenitors of white dwarfs, novae and type Ia supernovae, from those of core-collapse 
supernovae, neutron stars, and stellar mass black holes. The rate of the ${ }^{12} \mathrm{C}+{ }^{12} \mathrm{C}$ reaction, which is the trigger of the carbon burning, is a primary input to predict the behavior of a star at this branching point. Stellar models predict that quiescent carbon burning occurs for temperatures ranging between 0.5 and $1 \mathrm{GK}$, corresponding to center of mass energies between 0.9 and 3.4 $\mathrm{MeV}$. However, the larger the ${ }^{12} \mathrm{C}+{ }^{12} \mathrm{C}$ rate, the lower the temperature of the carbon burning. Consequently, also the duration of the $\mathrm{C}$ burning is modified by a variation of the ${ }^{12} \mathrm{C}+{ }^{12} \mathrm{C}$ rate. Carbon burning influences the energy generation and nucleosynthesis of massive stars. Actually, the two main channels of this reaction release protons and $\alpha$ particles in a rather hot environment, thus allowing a complex chain of reactions involving nuclei from $\mathrm{C}$ to $\mathrm{Si}$. The ${ }^{12} \mathrm{C}+{ }^{12} \mathrm{C}$ rate also affects the outcomes of type Ia supernovae which play a fundamental role in cosmology, allowing the measurements of distances and of the expansion rates of high redshift galaxies. In this context, energies as low as $0.7 \mathrm{MeV}$ become relevant.

The ${ }^{12} \mathrm{C}+{ }^{12} \mathrm{C}$ reaction, can proceed through different channels corresponding to the emission of a photon, a neutron, a proton, an $\alpha$ particle or even two $\alpha$ particles or a ${ }^{8} \mathrm{Be}$ nucleus. Of these channels, the two more relevant are the emission of protons and $\alpha$ particles. The Q-value for proton emission is $2.24 \mathrm{MeV}$ while that for $\alpha$ emission is $4.62 \mathrm{MeV}$. The proton and alpha channels can be measured either by detecting the charged particles or by revealing the gamma decay of the first excited state to the ground state of the ${ }^{23} \mathrm{Na}$ or ${ }^{20} \mathrm{Ne}$ residual nuclei, respectively. The energy of the two photons are $440 \mathrm{keV}$ for the proton channel and $1634 \mathrm{keV}$ for the alpha channel. Obviously, the gamma measurement cannot take into account the $\alpha_{0}$ and $\mathrm{p}_{0}$ (particles with the full energy which leave the residual nucleus in the ground state) as well as the contributions from high-energy states of the residual nuclei which de-excite directly to the ground state. Approximately, the decay of the first excited state to the ground state accounts for $50 \%$ of the total cross section. So far, many different experiments attempted to measure the ${ }^{12} \mathrm{C}+{ }^{12} \mathrm{C}$ reaction using one of the two above described techniques or both. The lowest energy measured is 2.1 $\mathrm{MeV}$ [8]. The general structure is characterized by the presence of several resonances superimposed on a flat background. The lowest energy resonance observed $(E=2.14 \mathrm{MeV})$ has a quite clear signature in the alpha channel but is unresolved in the proton channel due to the large uncertainties of the data at these energies. Nevertheless, it is characterized by a relative large strength and its impact on the reaction rate is very relevant. The measurement of the ${ }^{12} \mathrm{C}+{ }^{12} \mathrm{C}$ reaction is affected by beam induced as well as natural background issues. The former are primarily due to impurities in the carbon target, in particular $\mathrm{H}$ and ${ }^{2} \mathrm{H}$ because of their ease of forming bonds with carbon. These ions are also deposited on the surface of carbon targets from the vacuum rest gas during measurements. The latter affect the $\gamma$-ray spectrum and derive from naturally occurring sources, primarily ubiquitous natural radioisotopes. This background is negligible at higher energies, but becomes significant below 3.0 MeV. In the measurement of Spillane et al. [8], the HPGe detector was surrounded by a $15 \mathrm{~cm}$ thick lead shield allowing a reduction of the natural background by a factor of 400 near $\mathrm{E}=1.6 \mathrm{MeV}$. At LUNA, a proper massive shielding of $0.3 \mathrm{~m}^{3}$ of copper and lead, surrounded by an anti-radon envelope of Plexiglas flushed with $\mathrm{N}_{2}$ gas allowed a background suppression of 5 orders of magnitude for rays below $2 \mathrm{MeV}$ with respect to a background spectrum measured underground with no shielding [9]. Therefore, a deep underground measurement represents a unique possibility to reach the low energy domain of the ${ }^{12} \mathrm{C}+{ }^{12} \mathrm{C}$ reaction. The advantage of an underground measurement is less evident in the case of particle detection, even if a recent measurement performed at the LUNA 
$400 \mathrm{kV}$ accelerator proved that revealing low energy alpha particles is easier in a deep underground laboratory than overground [2]. At LUNA MV, The ${ }^{12} \mathrm{C}$ beam (single or double charged depending on the energy) will impinge on a solid ${ }^{12} \mathrm{C}$ target of natural composition with the as low as possible contamination due to hydrogen isotopes. Infinitely thick targets (e.g. $1 \mathrm{~mm}$ thick) are preferable since they enhance counting rates and are more resistant. As for the detection system, a high efficiency and ultra-low intrinsic background HPGe detector, as the one now in use at the LUNA $400 \mathrm{kV}$ accelerator [5,6,7], placed at 0 degrees with respect to the beam direction complemented with four silicon detectors (or telescopes) at backward angles will allow the measurement of both the $\gamma$-rays and the particles (protons and alphas). A search for low energy resonances with an HPGe detector will benefit of the low $\gamma$-ray background obtainable with a massively shielded setup underground. The possibility of using also silicon detectors/telescopes will allow to obtain a full picture of the reaction at least at the higher energies where particle detection will be easier. The measurement is expected to last about 2.5 years, assuming to explore the center of mass energy region down to 1.7 and $1.9 \mathrm{MeV}$ for the proton and alpha channel, respectively, with $5 \mathrm{keV}$ spacing to search for resonances with width of about $10 \mathrm{keV}$.

Finally, the LUNA MV scientific program foresee the measurement of the so-called neutron source reactions, ${ }^{13} \mathrm{C}(\alpha, \mathrm{n}){ }^{16} \mathrm{O}$ and ${ }^{22} \mathrm{Ne}(\alpha, \mathrm{n}){ }^{24} \mathrm{Mg}$. These are fundamental for the nucleosynthesis of almost half of the heavy elements $(\mathrm{A}>60)$ through the slow neutron capture process (s-process). More specifically, the cosmic creation of roughly half of all elements heavier than iron, including metals, such as $\mathrm{W}$ and $\mathrm{Pd}$, as well as rare earth, such as $\mathrm{La}$ and Nd, occurs in AGB stars thanks to the neutrons from ${ }^{13} \mathrm{C}(\alpha, n){ }^{16} \mathrm{O}$. As a consequence, the accurate and precise (at the level of $10 \%$ ) knowledge of the ${ }^{13} \mathrm{C}(\alpha, n){ }^{16} \mathrm{O}$ reaction is required at stellar temperatures in the range from 80 to 250 million $\mathrm{K}$. The current experimental situation does not fulfil such a requirement. In particular, the ${ }^{13} \mathrm{C}(\alpha, n){ }^{16} \mathrm{O}$ reaction $(\mathrm{Q}=2,216 \mathrm{MeV})$ has been studied over a wide energy range by several direct measurements (see e.g. [10]). Unfortunately, first of all there exist no data from direct measurements close to the energy of astrophysical interest because of the severe limitations imposed by the high neutron background in surface laboratories. Second, the lowest energy data are affected by uncertainties that are too large to constrain extrapolations of higher energy data to astrophysical energies. Finally, discrepancies exist between different data sets both in energy dependence and absolute values. LUNA will study ${ }^{13} \mathrm{C}(\alpha, n){ }^{16} \mathrm{O}$ in direct kinematics both with the $400 \mathrm{kV}$ accelerator and then with the 3.5 MV one. This way it will be possible to cover a wide energy range, to address the issue of normalization discrepancies and to minimize overall statistical and systematic uncertainties, taking advantage of the 3 orders of magnitude suppression of the laboratory neutron background of LNGS. In the energy region of interest, $\mathrm{E} \alpha=0.3-1.4$ $\mathrm{MeV}$ neutrons are emitted with energies $\mathrm{E}_{\mathrm{n}}=2.0-3.5 \mathrm{MeV}$ thus requiring moderation before detection. A likely geometry of the detector will consist of about $20{ }^{3} \mathrm{He}$ low activity tubes embedded in a moderating polyethylene matrix. From a realistic estimate of the signal and of the background it seems possible to access the center of mass energy region down to $230 \mathrm{keV}$.

The ${ }^{22} \mathrm{Ne}(\alpha, \mathrm{n})^{25} \mathrm{Mg}$ is the main source of neutrons in AGB stars of initial masses higher than roughly 4 solar masses. In such stars elements like $\mathrm{Rb}$ and $\mathrm{Zr}$ are formed. Also for AGB stars of lower initial masses (about 3 solar masses), where the ${ }^{13} \mathrm{C}(\alpha, n){ }^{16} \mathrm{O}$ reaction is the main neutron source, it has been shown that the ${ }^{22} \mathrm{Ne}(\alpha, \mathrm{n})^{25} \mathrm{Mg}$ neutron burst impacts the abundances of almost 200 nuclei [11]. Finally, the ${ }^{22} \mathrm{Ne}(\alpha, \mathrm{n})^{25} \mathrm{Mg}$ reaction is also the main neutron source for the s- 
process occurring during the hydrostatic burning of massive stars (with initial mass greater than 10 solar masses) [12] which is responsible for the cosmic production of the elements between the Fe peak and $\mathrm{Sr}$. The current knowledge of the ${ }^{22} \mathrm{Ne}(\alpha, \mathrm{n})^{25} \mathrm{Mg}$ reaction is incomplete and imprecise: the rate is given with an uncertainty of 20-30\% while less than 5\% is required for accurate model predictions. LUNA MV will study ${ }^{22} \mathrm{Ne}(\alpha, \mathrm{n})^{25} \mathrm{Mg}$ with a ${ }^{4} \mathrm{He}$ beam on a windowless gas target of enriched ${ }^{22} \mathrm{Ne}$ surrounded by a $4 \pi$ neutron detector, most likely the same designed to be used for the ${ }^{13} \mathrm{C}(\alpha, n){ }^{16} \mathrm{O}$ study. From an estimate of signal (in absence of resonance) and background, it looks feasible to have an accurate cross section measurement down to the center of mass energy of $620 \mathrm{keV}$. At lower energies, it will be possible to exclude any resonance able to give a significant contribution to neutron production in AGB stars.

As of today, the LUNA MV accelerator will be delivered at LNGS at the end of 2018. The following six months will be dedicated to the commissioning of the machine and its energy calibration. Therefore, the scientific program above described will start in the second half of 2019 and last 5-6 years at least. The flagship reaction for the future program will be the ${ }^{12} \mathrm{C}(\alpha, \gamma){ }^{16} \mathrm{O}$, that determines the helium burning time scale and, together with the convection mechanism, the abundances of carbon and oxygen at the end of helium burning. The carbon abundance at the end of helium burning has important consequences for the subsequent evolution of the star and for the nucleosynthesis path. Stellar models show an exceptional sensitivity on the cross section of ${ }^{12} \mathrm{C}(\alpha, \gamma){ }^{16} \mathrm{O}$ and an experimental determination with a precision of the order of $10 \%$ or better is required to provide adequate constraints on stellar evolution.

\section{References}

[1] C. Iliadis, Nuclear Physics of Stars, Wyley Weinheim, 2007

[2] C. G. Bruno et al., Eur. Phys. J A 51 (2015) 94

[3] U. Greife et al., Nucl. Instr. and Meth. A 350 (1994) 327

[4] A. Formicola et al, Nucl. Instr. and Meth. A 507 (2003) 609

[5] H. Costantini et al., Rep. on Prog. in Phys. 72 (2009) 086301/1

[6] C. Broggini et al., LUNA: Ann. Rev. of Nucl. and Part. Sci. 60 (2010) 53

[7] Topical Issue ñUnderground nuclear astrophysics and solar neutrinos: Impact on astrophysics, solar and neutrino physicsò Eur Phys. J A 52 (2016)

[8] T. Spillane et al., Phys. Rev Lett. 98 (2007) 122501

[9] A. Caciolli et al., Eur. Phys. J A 39 (2009) 179

[10] M. Heil et al., Phys. Rev C 78 (2008) 025803

[11] S. Bisterzo et al., MNRAS 449 (2015) 506

[12] M. Pignatari et al., Astrophys. J. 710 (2010) 1557 